\title{
Large Meningomyelocele Defects Reconstruction Using Double Opposing Fasciocutaneous Flaps
}

\author{
Durga Prasada Rao Pothula ${ }^{1}$ \\ ${ }^{1}$ Department of Plastic Surgery, Rangaraya Medical College, Kakinada, Andhra Pradesh, India.
}

\section{ABSTRACT}

\section{BACKGROUND}

When a part of neural tube fails to develop or close properly, it manifests as spina bifida. Spina bifida defects range from the mild form (spina bifida occulta) to severe form (meningomyelocele). Meningomyelocele is to be closed surgically as early as possible to prevent infection and other complications. Many methods are available for treatment of meningomyelocele defects ranging from skin graft to flap cover. Meningomyelocele defects when small in size can be closed primarily. Large meningomyelocele defects poses technical challenge to reconstructive surgeon. Many flap cover techniques were described to cover the large meningomyelocele defects. We wanted to evaluate bilateral opposing fasciocutaneous flaps method for closure of large defects without tension.

\section{METHODS}

To cover large meningomyelocele defects, fasciocutaneous flaps give good and stable cover, and prevents wear and tear, and infections. We have used double opposing fasciocutaneous flaps method for reconstruction of large meningomyelocele defects. The advantages of this technique are its simplicity and it being a single stage procedure. 20 children with meningomyelocele defects of various sizes were operated with this method and followed for 6 months for wound dehiscence and CSF leak.

\section{RESULTS}

Closure of large meningomyelocele defects is made possible with simple and easy technique. Ease of planning and execution even for the inexperienced are the most noticeable features. All children were well post operatively. Two patients in whom flaps were taken with acute angles suffered tip necrosis. All flaps gave stable cover to the defect without dehiscence at the suture line. The entire reconstructive process consumed less time and was without major complications.

\section{CONCLUSIONS}

Neural tube malformation congenitally causes spina bifida defects. Spina bifida cases may range from simple to severe defects. Treatment of meningomyelocele spans from primary closure to flap cover closure. Closure of large meningomyelocele defect by bilateral fasciocutaneous flaps is a good method to adopt. This method is simple, less time consuming, technically non-demanding and easily reproducible.

\section{KEY WORDS}

Meningomyelocele, Double Opposing Fasciocutaneous Flaps, Tension Free Closure
Corresponding Author:

Durga Prasada Rao Pothula, Assistant Professor,

Department of Plastic Surgery,

Rangaraya Medical College,

Kakinada-533003,

Andhra Pradesh, India.

E-mail: pothuladp@gmail.com

DOI: $10.14260 /$ jemds/2020/250

Financial or Other Competing Interests: None.

How to Cite This Article:

Pothula DPR. Large meningomyelocele defects reconstruction using double opposing fasciocutaneous flaps. J. Evolution Med. Dent. Sci. 2020;9(14):1152-1155, DOI: $10.14260 /$ jemds/2020/250

Submission 06-011-2019,

Peer Review 01-01-2020,

Acceptance 09-01-2020,

Published 06-04-2020.

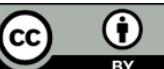




\section{BACKGROUND}

Neural tube is the embryonic structure that eventually develops into the baby's brain, the spinal cord and the tissues that enclose them. Formation of the neural tube starts early in the pregnancy and gets closed by $28^{\text {th }}$ day after conception. In children with spina bifida a portion of the neural tube fails to develop or close properly. Spina bifida can range from mild to severe and depends on type of defect, size and location. Neural tube defects, the non-fusion of embryonic neural arches may range from spina bifida occulta to meningomyelocele. ${ }^{1}$ Neural tube defects can affect any part of neuroaxis. The main defect is a failure of closure of the caudal neuropore. Meningomyelocele is a congenital defect where in embryonal formation of neural tube is at defect leading to protrusion of malformed spinal cord, meninges through incompletely closed vertebral arches. ${ }^{2}$ And forms a sac filled with CSF. Skin over the sac is at defect and is covered with a thin layer. Spina bifida occulta results in a small separation or gap in one or more vertebrae. Usually it goes unrecognised and is discovered during imaging for some other cause. Meningocele is a form of spina bifida where in spinal cord is intact and is within the spinal canal but the meninges bulges out of vertebrae forms a as ac filled with CSF.

Spina bifida with meningomyelocele is more common and the most severe form of all. ${ }^{3}$ The vertebral column defects include lack of fusion of the vertebral arches, laterally displaced pedicles and a widened spinal canal. The spinal cord instead of forming a tube, the neural folds persist as a flat plate of tissue called as neural placode. ${ }^{4}$ This condition causes neural dysfunction and paralysis below the level of the lesion. Some cases are associated with calvarial defects. ${ }^{5}$

Children with meningomyelocele are born with large exteriorised sac covered with delicate skin. It may be associated with severe dysfunction of the cauda equine roots or conus medullaris contained in the sac. In such a situation, both the lower limbs are paralytic, urinary bladder and bowel dysfunction leading to urine and faecal continence problems. Meningomyelocele patients may be associated with hydrocephalus and a Chiari II malformation. ${ }^{6}$

The exact etiological factors are not known but some risk factors are identified. Folic acid deficiency is one of such main risk factors. Its deficiency increases the risk of spina bifida and other neural tube defects. Family history of neural tube defects is another risk factor. A woman having spina bifida defect will have more chances to give birth to a child with spina bifida. Parents having one more children with spina bifida have more chances to give birth to a child with spina bifida. Medications like antiepileptic drugs like Valproic acid seems to cause spina bifida if taken during pregnancy. Women with Diabetes with poor glycaemic control will have higher chances to give birth to spina bifida babies. Other medical comorbid conditions like obesity, hyperthermia during pregnancy are some of the risk factors.

The complications of Spina bifida may range from very minimal to severe. Severity depends upon by the size and location of the neural tube defect, whether the skin is intact at the affected site, whether the spinal cord is affected and comes out of the defect. If the nerves that supply lower limb gets affected leads to weakness of muscles and paralysis. This later leads to walking and ambulatory functions problems. The problems like scoliosis, other problems of muscular involvement appears as some of the orthopaedic complications. If the involved segment of spinal cord supplies bowel and bladder, their function gets affected. Accumulation of CSF and formation of hydrocephalus may complicate babies with meningomyelocele. Chiari Malformation II is a brain abnormality associated with meningomyelocele. There are likely chances of getting infected of the meninges leading to meningitis. Tethering of spinal cord where in the cord with nerves gets attached to the scar tissue of the defect is one of the complication and may ensue post operatively also at repair site.

When left untreated virtually all die within the first year and aggressive treatment had a profound effect on survival and quality of life. While early surgical management is the protocol practised widely unless the child is sick. The treatment is aimed at decompression and closure of neural tube, dura and skin without tension.

Wound closure can be achieved through primary closure, skin grafting or flap cover depending upon the size of the defect.6,7 Small defects can be closed primarily and larger defects needs fasciocutaneous flap cover for stable closure. We have repaired large meningomyelocele defects in 20 children using double opposing fasciocutaneous flap cover. This technique is simple, less time consuming and easy to reproduce.

\section{Objective}

We wanted to evaluate bilateral opposing fasciocutaneous flaps method for closure of large defects without tension.

\section{METHODS}

Data of Children having meningomyelocele who were operated using double opposing fasciocutaneous flaps technique was analysed and formed for retrospective descriptive study. Children are of age group from 14 days to 2 months were undergone surgery with mean of 17 days. Children's parents were explained of the nature of surgery for closure of defect that aims at prevention of complications rather than curing the neurological deficit. And informed consent was taken from the parents mentioning the same. Institutional Ethics committee approval was taken to study these children retrospectively. 20 Children were studied retrospectively for closure of large meningomyelocele defect. Combinations of paraplegia, bladder and bowel dysfunction are present in all the children. After clinical evaluation all the children were subjected to ultrasonographic examination. Magnetic Resonance Imaging is done to evaluate the neurological deficits. The size of meningomyelocele defect ranges from $28 \mathrm{Sq} \mathrm{cm}$ to $56 \mathrm{Sq} \mathrm{mm}$.

\section{Surgical Technique}

After intubation, the child is made to lie in prone position. After routine surgical draping, neurosurgery team initiates the surgery. Careful debridement and decompression done on spinal cord. Spinal cord repositioned back into spinal canal. Dura is closed aiming at leak free closure. 


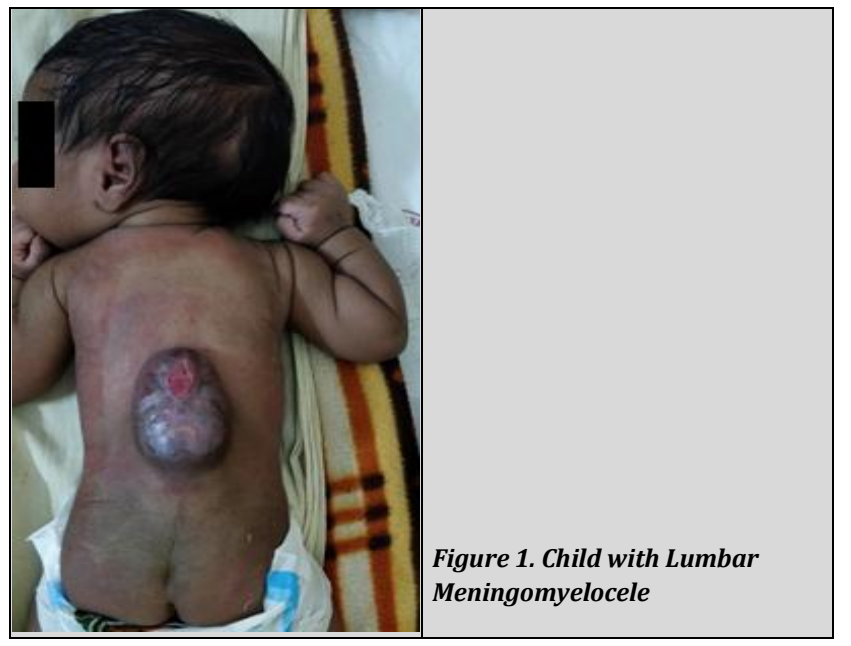

\begin{tabular}{|c|c|c|}
\hline Sl. No. & Size of Defect & No. of Children \\
\hline 1. & 30 Sq. $\mathrm{Cm}$ & 9 \\
\hline 2 & $31-40 \mathrm{Sq} \mathrm{Cm}$ & 6 \\
\hline 3. & 41-50 Sq. Cm & 3 \\
\hline 4. & 51-60 Sq. Cm & 2 \\
\hline \multicolumn{3}{|c|}{ Table 1. Size of Defects after Decompression of Neural Elements } \\
\hline Sl. No. & Complications & No. of Children \\
\hline 1. & Tip necrosis & 2 \\
\hline 2. & CSF leak & 3 \\
\hline 3. & Total flap loss & Nil \\
\hline 4. & Wound dehiscence & Nil \\
\hline 5. & Post-Operative infections & Nil \\
\hline \multicolumn{3}{|c|}{ Table 2. Statistics of Complications } \\
\hline
\end{tabular}
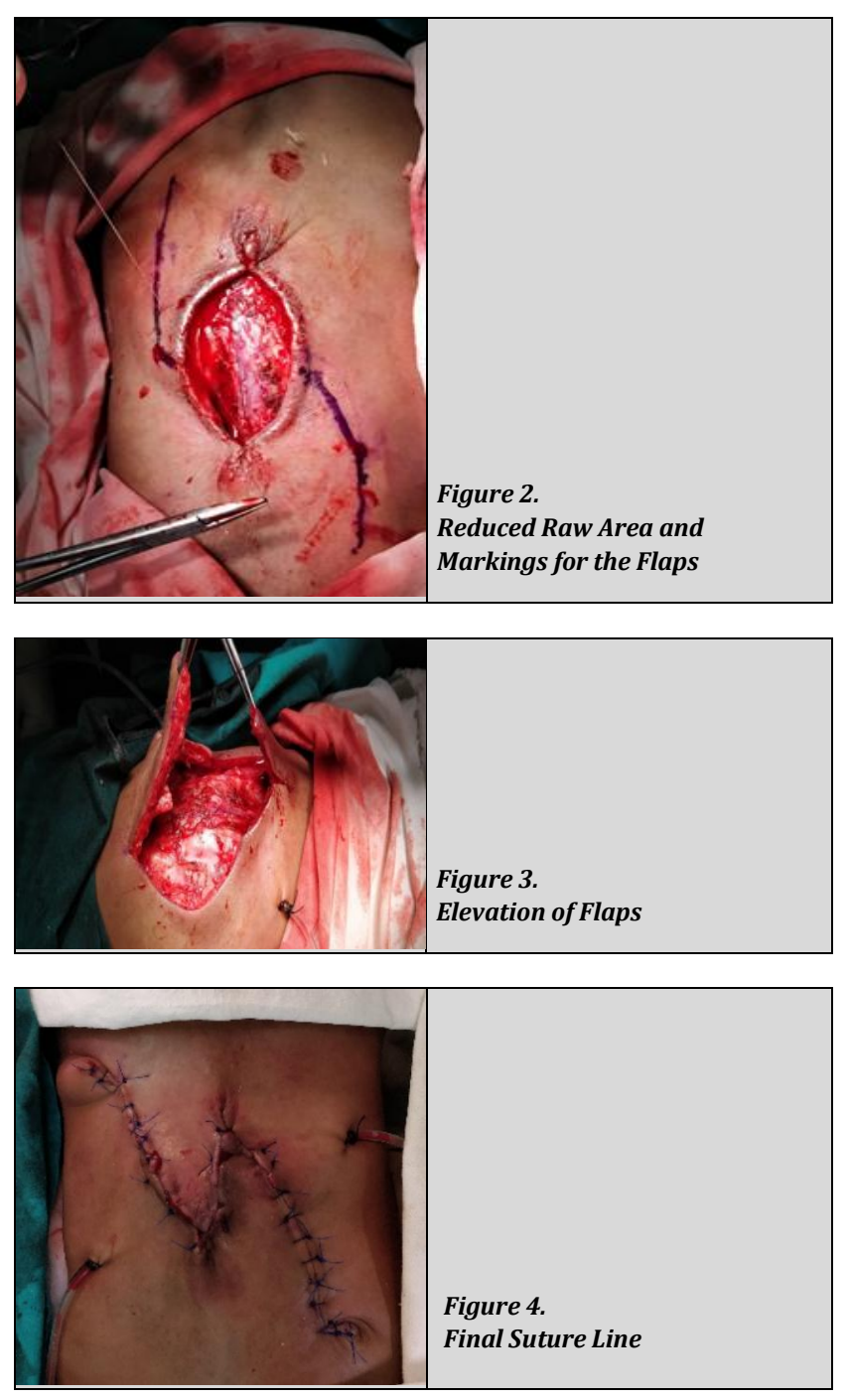

Unhealthy skin over the lesion is debrided until healthy skin margins obtained. The resultant defect which appears large is reduced to possible extent by suturing at superior and inferior corners on vertical axis (fig. 1). Marking for the flaps is done starting at the middle of the vertical margins on either side of the defect (fig. 2). Avoiding acute angles markings are done in such way the lines follow vertical axis but with minimal curvature. The maximum width of the flap from the margin of the defect should be more than the half of the defect at its maximum width. Flaps are raised with fascia through plane of fat underneath. Undermining to the necessary extent is done at surrounding skin to ensure tension free suturing. Flaps inset given and two layered suturing done. $1^{\text {st }}$ layer with monofilament absorbable and $2^{\text {nd }}$ layer with polypropylene. Tube drains are kept on either side of the repair without suction devices.

Post operatively the child was nursed in prone and lateral positions avoiding supine position. Drains were removed on noticing no drainage or the downward trend of drainage fluid. All the children were followed for 6 months.

\section{RESULTS}

All the patients were repaired of the defect healed well without dehiscence of suture line. The average duration of surgery is less than one hour. Two of the patients developed tip necrosis of flaps noticed on $2^{\text {nd }}$ post-operative day and were debrided and all were healed well secondarily. Later this was avoided by adopting obtuse angled flaps. Three of the children had CSF leak through drainage tubes for 5-7 days later stopped spontaneously. No complete loss of flaps or wound dehiscence in noticed.

\section{DISCUSSION}

Risk of spina bifida can be significantly be reduced by adding Folic acid to the diets of child bearing age women. Foods that contain Folic acid are cereals, green leafy vegetables egg yolks. Women should start using folic acid before they consider becoming pregnant. Using Valproate be better avoided during pregnancy and women with diabetes should aim at good glycaemic control to have the minimised risk of spina bifida. Controlling obesity, proper of hyperthermic condition of various medical conditions keeps the risk of spina bifida at minimal level. Treatment of spina bifida depends on type and severity of the disorder.

There is no definitive cure for spina bifida. Damaged nerve tissue cannot be repaired nor the functions can be restored. Mild form of spina bifida doesn't require any surgery. Other varieties require appropriate surgical repair. Foetal surgery is done in utero to repair spina bifida. It is aimed at closing the abnormal opening on the developing neuroaxis. The access is gained through mother abdomen and opening the uterus. Foetal surgery is beneficial in early closure of the defect and avoids contact of neuronal tissue with amniotic fluid, minimises the risk of formation of Chiari malformation II.

Meningomyelocele defect closure can be accomplished through primary closure, skin grafting and flap cover. Closure is aimed at stable cover to prevent infection and proper cover 
for neuronal elements and to protect exposed neuronal tissue and their cover from additional trauma ${ }^{3}$. Small defects can be closed by approximating the skin flaps with relaxation by undermining. Majority of the meningomyelocele defects are amicable for primary closure. Large meningomyelocele defects where direct closure is not possible and needs adaptation of other mode of reconstructive procedure for closure. Forceful attempts for primary closure may cause necrosis of flaps, wound dehiscence and infection. Many methods have been described for closure of large meningomyelocele defects ranging from skin grafts to flap cover. Majority of children suffer from hydrocephalus where in there is accumulation of CSF in subarachnoid space. Most of these children requires ventricular drainage shunt to prevent accumulation of CSF. After the definitive surgical repair to cover the defect is done, other surgical procedures may be needed to correct the deformities of spine, foot and hip.

Covering with skin graft has the advantage of being simple procedure and no meticulous planning is required. And skin grafting carries the disadvantage of providing unstable cover, gives up and cracks are formed on long term wear and tear. And also doesn't provide soft cushion like tissue over the neuronal tissue. ${ }^{8}$ So flaps are preferred to skin grafting for closure of meningomyelocele defects.

Many local flaps are described like transposition, rotation, bilateral V-Y, bilobed, Limberg flaps. The Limberg flap is more useful for medium sized defects and tension free closure not ensured for larger defects. ${ }^{9}$ Latissimus dorsi and gluteal myocutaneous flaps are also useful to cover the defects but carries the drawback of longer operating time and more bleeding and chances of disturbing the musculoskeletal activity in future. ${ }^{10}$ Covering the large meningomyelocele defects with fasciocutaneous flaps is good options as it gives stable cover of the defect. Bilateral fasciocutaneous flap method is having advantages of being simple, easily adoptable, shorter operating time. And the donor areas are closed primarily. Though it is preferable to have suture line away from dural closure suture, the bilateral fasciocutaneous flaps method gives good and stable healing of suture line. Barring suture line that comes over the suture line of dural closure, the flaps healed well giving sable cover of the defect. And in this procedure muscles are not disturbed and so no functional compromise at shoulder.

\section{CONCLUSIONS}

Spina bifida is a congenital defect due to neural tube malformation. The defects due to spina bifida cases may range from simple to severe defects. Early surgical closure of the defect is needed to prevent complications. Treatment of meningomyelocele spans from primary closure to flap cover closure. Closing the large defects with fasciocutaneous flaps gives stable cover. Closure of large meningomyelocele defects by bilateral fasciocutaneous flaps is a good method to adopt. Large meningomyelocele defects can be closed easily with bilateral fasciocutaneous flaps. This method technically is nondemanding, simple, less time consuming, easy to plan, and execute technique and results in minimal bleeding. In our study there is no dehiscence of suture line upon follow up for 6 months.

\section{REFERENCES}

[1] Moore KL, Persaud TVN, Torchia MG. The developing human. $9^{\text {th }}$ edn. Elsevier 2013: p. 398.

[2] Harms J. www.harms-spinesurgery.com 2007.

[3] El-Khatib HA. Large thoracolumbar meningomyelocele defects: Incidence and clinical experiences with different modalities of latissimus dorsi musculocutaneus flap. Br J Plast Surg 2004;57 (5):411-17.

[4] Townsend CM, Beauchamp RD, Evers BM, et al. Sabiston text book of surgery. The biological basis of modern surgical practice. Vol. 2. 17th edn. New York: Elsevier Saunders 2004: p. 2171-2.

[5] Shaer CM, Chescheir N, Schulkin J. Myelomeningocele: a review of the epidemiology, genetics, risk factors for conception, prenatal diagnosis, and prognosis for affected individuals. Obstet Gynecol Surv 2007;62 (7):471-9.

[6] Ozcelik D, Yildiz KH, Iş M, et al. Soft tissue closure and plastic surgical aspects of large dorsal myelomeningocele defects (review of techniques). Neurosurg Rev 2005;28 (3):218-25.

[7] Hexsel CL, Loosemore M, Goldberg LH, et al. Postauricular skin: an excellent donor site for split-thickness skin grafts for the head, neck, and upper chest. Dermatol Surg 2015;41 (1):48-52.

[8] Patterson TJ. The use of rotation flaps following excision of lumbar meningomyeloceles: an aid to the closure of large defects. Br J Surg 2005:54:640-4.

[9] Lapid 0, Rosenburg L, Cohen A. Meningomyelocele reconstruction with bilobed flaps. Br J Plast Surg 2001;54 (7):570-2.

[10] Luce EA, Walsh J. Wound closure of the myelomeningocoele defect. Plast Reconstr Surg 1985;75 (3):389-93. 